\title{
PERCEPCIONES PARENTALES SOBRE EL IMPACTO DEL SÍNDROME DE DOWN EN LA FAMILIA
}

\section{Parental perceptions of the impact of Down syndrome in the family}

\author{
Laura SERRANo FERnÁNDEZ \\ Colegio de Educación Especial de Cambrils. Madrid \\ Avenida Ciudad de Barcelona, 138, Escalera 3, 5. ${ }^{\circ} \mathrm{D}$ \\ 28007 Madrid \\ lauraserfer@gmail.com \\ Dolores IZUZQuiza GASSET \\ Universidad Autónoma de Madrid. Facultad de Formación de Profesorado y Educación
}

Recepción: 29 de enero de 2017

Aceptación definitiva: 7 de julio de 2017

Resumen: La parentalidad de un hijo con síndrome de Down supone un desafío, no sólo para los padres, sino también para el conjunto del sistema familiar. La presente investigación se propone analizar, desde un punto de vista cualitativo, la visión que los propios padres tienen sobre el impacto, tanto positivo como negativo, que la presencia de un hijo con SD provoca en la familia. Para ello, se han analizado las entrevistas realizadas a 10 participantes ( 3 padres, 3 madres y 4 profesionales del ámbito de la educación especial). Los resultados revelan que, a pesar de que el diagnóstico del SD en un hijo sea un evento inesperado y estresante para la familia, la percepción general del impacto familiar es positiva.

Palabras Clave: síndrome de Down; impacto familiar; familia; discapacidad intelectual.

AbstRact: Parenting a child with Down syndrome is a challenge, not only for parents, but also for the whole family system. The present research aims to analyse, from a 
qualitative point of view, the vision that the parents themselves have of the impact, both positive and negative, that the presence of a child with DS causes in the family. To this end, interviews with 10 participants ( 3 parents, 3 mothers and 4 professionals in the field of special education) have been analysed. The results reveal that, although the diagnosis of SD in a child is an unexpected and stressful event for the family, the general perception of the family impact is positive.

KEY wORDs: Down syndrome; family impact; family; intellectual disability.

\section{Introducción}

$\mathrm{E}$

L SínDROME DE Down (de ahora en adelante, SD), originado en el 95\% de los casos por la trisomía 21 (Sherman, Allen, Bean y Freeman, 2007), está considerado como la aneuploidía más frecuente de cromosomas autosómicos y la principal causa genética de discapacidad intelectual en el ser humano (Mullins et al., 2013). Actualmente, muestra una prevalencia de 1 de cada 800 nacimientos, lo que se traduce en aproximadamente entre 5 y 8 millones de casos a nivel mundial (Lancet Neurology, 2016). Aunque la característica fundamental del síndrome es la presencia de discapacidad intelectual en grados variables, el SD manifiesta con alta frecuencia una serie de trastornos comórbidos que requieren atención sanitaria (Arumugam et al., 2015).

La comunicación del diagnóstico del SD en un hijo se constituye como el primer evento de impacto familiar de dicha discapacidad, siendo el concepto de impacto familiar definido por Stein y Riessman (1980) como el efecto multidimensional que provoca una condición vital crónica de uno de los miembros en el grupo familiar. Este evento, considerado de transcendental importancia para las familias, se caracteriza por encontrarse dominado por emociones devastadoras como consecuencia de un acontecimiento siempre inesperado (Greiner y Conklin, 2015). La primera noticia del diagnóstico de la discapacidad se consolida como un período de agitación en el que surgen sentimientos de tristeza (Pereira-Silva, Oliveira y Rooke, 2015), pérdida, dolor, incertidumbre y amenaza (Federación Estatal de Asociaciones de Profesionales de la Atención Temprana-GAT, 2011), además de confusión y estrés (Collins y Coughlan, 2016). Se puede afirmar que el hecho de la parentalidad de un hijo con SD u otro tipo de discapacidad, frente a la crianza de un hijo de desarrollo típico, es asumido como un reto específico que los padres deben afrontar de manera individual (Migerode, Maes, Buysse y Brondeel, 2012). En consonancia con la perspectiva holística e integradora de la Teoría General de Sistemas (Von Bertalanffy, 1968), la familia debe ser concebida como un conjunto social de miembros independientes aunque interrelacionados entre sí, por lo que aquello que le ocurre a uno de los integrantes del sistema repercute de forma variable en los demás individuos (Turnbull y Turnbull, 2001). De este modo todos los individuos constituyentes del sistema se enfrentan al reto de la discapacidad generando, como consecuencia, una cadena de demandas específicas (Migerode et al., 2012), clasificadas, según criterios establecidos por Almasri et al. (2011), en demandas asociadas a la condición específica del individuo, demandas 
vinculadas al funcionamiento familiar y demandas referentes a la vida en comunidad y a los recursos económicos.

Hasta la década de los 80 , la parentalidad de un hijo con discapacidad se asociaba con la aparición de efectos adversos que perturbaban el correcto funcionamiento del sistema conyugal y familiar (Farber y Kirk, 1959; Gath, 1977; Kramm, 1963; Schonell y Watts, 1956). No obstante, a partir de los años 80 comienza a proliferar literatura focalizada en aportar una visión más optimista que pretende abordar los efectos positivos de la vivencia de la discapacidad en el seno familiar (Helff y Glidden, 1998; Hornby, 1995), haciendo énfasis en el afianzamiento de los vínculos parentales y filiales o el incremento de los niveles cohesivos de la estructura familiar (Glidden, Bamberger, Turek y Hill, 2010; Green, 2007; Hastings y Taunt, 2002). En SD se habla, incluso, de la existencia de una cierta ventaja frente a discapacidades de diversas etiologías (Mitchell, Hauser-Cram y Crossman, 2015). Las características positivas de la expresión fenotípica del SD, el afable perfil estereotipado de su personalidad, la alta tasa de incidencia, la posibilidad de diagnóstico prenatal y el incremento de su aceptación social ayudan a corroborar esta idea de ventaja (Adamson, Deckner y Bakeman, 2010; Mitchell et al., 2015). Mientras, factores como el mayor nivel de trastornos de salud o la presencia de importantes alteraciones conductuales, características propias de otras clases de trastornos genéticos o del desarrollo, conducen a la idea de una cierta desventaja frente a discapacidades como el síndrome de Down (Lanfranchi y Vianello, 2012).

En términos generales, las familias con hijos con SD presentan una sensación de normalidad (Cunningham, 1996), mientras que sus miembros se autoperciben de forma positiva, puntualizando que la persona con SD que vive con ellos es feliz (Durmaz et al., 2011). Sin embargo, y a pesar de que se considere que la impresión general de las familias con miembros con SD es de normalidad, resulta evidente que el impacto de acoger a un hijo que se sale de la norma provoca en la familia un cambio de organización y redefinición de sus roles, implicando una serie de necesidades funcionales merecedoras de futuros análisis exhaustivos (Albuquerque, Pereira, Fonseca y Canavarro, 2012).

\section{Método}

\subsection{Diseño}

El estudio mantiene un diseño en base a la tradición cualitativa y, concretamente, en base al enfoque de la teoría fundamentada. Este enfoque se basa en la generación de forma inductiva de una teoría a partir de los datos recogidos, sin partir de una hipótesis inicial, para lo que se realiza un proceso de codificación en el cual se construyen categorías para los datos, se determinan las relaciones existentes entre ellas y se conceptualizan las mismas en un nivel superior de abstracción. El objetivo primordial se focalizó en la comprensión de la estructura fenomenológica de cómo las familias perciben el impacto positivo y negativo que el SD provoca en el sistema familiar, para lo que el instrumento utilizado ha sido la entrevista semiestructurada 
con familias y profesionales educativos. Con el objetivo de asegurar la fiabilidad de los datos obtenidos, estos fueron sometidos a un proceso heurístico de triangulación entre los datos aportados por el grupo de padres y madres de hijos con SD y por el grupo de profesionales, comparando, de este modo, las visiones de ambos colectivos sobre las mismas cuestiones.

\subsection{Participantes}

Los participantes en el estudio cumplen con los requisitos de accesibilidad, idoneidad y representatividad, seleccionados a través de un muestreo intencional en base a los siguientes requisitos: un grupo A, conformado por padres y madres con al menos un/a hijo/a con síndrome de Down en edad escolar, y un grupo B, constituido por profesionales del ámbito de la educación especial. Se tomó una muestra total de 10 sujetos en el Colegio Concertado de Educación Especial Cambrils, situado en Madrid capital.

El grupo A está compuesto por 3 padres y 3 madres, con edades comprendidas entre los 42 y los 56 años, todos ellos casados con personas ajenas a la muestra. En cuatro de los casos, los individuos tienen estudios superiores, mientras que en los dos restantes han superado los estudios primarios y secundarios respectivamente. Las edades de los hijos con SD comprenden diferentes etapas del desarrollo con edades entre 7 y 21 años. Todas las familias cuentan con más de un hijo, siendo cuatro los casos con dos hijos, una familia con siete y otra de ellas con once, no existiendo, por tanto, ningún caso de hijo único.

\begin{tabular}{|c|c|c|c|c|c|c|c|c|}
\hline & $\begin{array}{l}\text { CÓDIGO } \\
\text { (P)ADRE } \\
\text { (M)ADRE }\end{array}$ & EDAD & $\begin{array}{c}\text { GÉNERO } \\
\text { (F)EMENINO } \\
\text { (M)ASCULINO }\end{array}$ & $\begin{array}{c}\text { ESTADO } \\
\text { CIVIL }\end{array}$ & $\begin{array}{l}\text { NIVEL DE } \\
\text { ESTUdIOS }\end{array}$ & $\begin{array}{c}\text { EDAD DEL } \\
\text { HIJO } \\
\text { AÑO + MES }\end{array}$ & $\begin{array}{c}\text { CONDICIÓN } \\
\text { DEL HIJO }\end{array}$ & $\begin{array}{c}\text { POSICIÓN } \\
\text { DEL HIJO SD } \\
\text { EN LA FAMILIA }\end{array}$ \\
\hline \multirow{6}{*}{$\begin{array}{c}\text { Grupo } \\
\text { A }\end{array}$} & P1 & 50 & M & Casado & Superiores & $7+10$ & SD & $1 / 2$ \\
\hline & P2 & 47 & M & Casado & Superiores & $8+10$ & SD & $2 / 2$ \\
\hline & $\mathrm{P} 3$ & 42 & $\mathrm{M}$ & Casado & Superiores & $7+7$ & SD & $2 / 2$ \\
\hline & M1 & 47 & $\mathrm{~F}$ & Casada & Superiores & $12+3$ & SD & $7 / 7$ \\
\hline & M2 & 56 & F & Casada & Primarios & $21+6$ & SD & $2 / 2$ \\
\hline & M3 & 46 & $F$ & Casada & Secundarios & $21+1$ & SD & $3 / 11$ \\
\hline
\end{tabular}

El grupo B está conformado por cuatro profesionales del ámbito de la educación especial, concretamente dos logopedas y dos maestras-tutoras. El grupo está formado en su totalidad por mujeres, con estudios superiores dentro de su campo profesional. Sus edades oscilan entre los 28 y los 53 años y con una experiencia profesional de entre 2 y 15 años en el mismo centro laboral. 
TABLA 2. Datos sociológicos del grupo B de la muestra (Fuente: Autor, 2017)

\begin{tabular}{|c|c|c|c|c|c|c|}
\hline & $\begin{array}{c}\text { CÓDIGO } \\
\text { (M)AESTRA } \\
\text { (L)OGOPEDA }\end{array}$ & EdAd & $\begin{array}{c}\text { GÉNERO } \\
\text { (F)EMENINO }\end{array}$ & $\begin{array}{c}\text { CATEGORía } \\
\text { DE ESTUDIOS }\end{array}$ & $\begin{array}{c}\text { CARGO } \\
\text { EN EL CENTRO }\end{array}$ & $\begin{array}{c}\text { EXPERIENCIA } \\
\text { EN EL PUESTO } \\
\text { (AÑOS) }\end{array}$ \\
\hline \multirow{3}{*}{$\begin{array}{c}\text { Grupo } \\
\text { B }\end{array}$} & MT1 & 49 & F & Superiores & Maestra-tutora & 2 \\
\cline { 2 - 7 } & MT2 & 28 & F & Superiores & Maestra-tutora & 6 \\
\cline { 2 - 7 } & L2 & 29 & F & Superiores & Logopeda & 7 \\
\hline
\end{tabular}

\subsection{Procedimiento}

La recolección de datos se llevó a cabo mediante la realización de entrevistas semiestructuradas de aproximadamente una hora de duración, durante los meses de febrero y marzo del año 2016, realizándose todas ellas en la sede del Colegio Concertado de Educación Especial Cambrils, excepto dos de las mismas que, por motivos de facilitación al acceso y participación de la muestra, se llevaron a cabo en el domicilio particular de los informantes. Las entrevistas fueron grabadas previo consentimiento escrito de los participantes y conducidas por una figura profesional experta en activo laboral, siendo su campo de actuación profesional y personal ajeno a la muestra para garantizar el factor de objetividad. Previamente a cada una de las sesiones, los participantes fueron informados, a través de una carta, sobre el objetivo del estudio, mientras que en el momento de la sesión se les entregó un acuerdo escrito de confidencialidad de los datos e información recabada.

El análisis de datos se llevó a cabo en tres etapas: 1) Análisis cualitativo de categorización y codificación de la información recogida en las entrevistas parentales. 2) Confrontación y triangulación de datos entre las entrevistas parentales y profesionales. 3) Generación de la teoría fundamentada.

Como soporte analítico se utilizó el software de análisis visual de datos cualitativos Atlas.ti, versión 7.5.2, para la determinación de redes estructurales y la consiguiente elaboración de la teoría general en base a la categorización y codificación de la información.

\section{Resultados}

A partir de la transcripción de las entrevistas semiestructuradas se realiza una primera codificación de las categorías conceptuales en las que se puede agrupar la información aportada por los participantes (codificación abierta), generando un total de 17 códigos. La selección y agrupación de estos códigos se ha realizado a partir del análisis de la información contenida en las entrevistas, para lo cual se ha utilizado la herramienta Atlas.ti, que permite seleccionar los conceptos contenidos en las entrevistas y ordenarlos en función del número de veces que se repiten en la entrevista, a partir de 
lo cual se realiza un proceso de agrupación y análisis hasta determinar las categorías principales. Paralelamente se realizan mapas de redes estructurales que permiten visualizar las relaciones existentes entre los diferentes códigos generados. Las relaciones encontradas entre conceptos nos permiten agregar los códigos en un nivel superior de abstracción, agrupándolos en cuatro categorías auxiliares ("dominio personal de la condición de SD”, "vivencia personal y emocional”, "experiencia familiar y social” e “impacto económico”), que a su vez se estructuran en torno a una categoría central, "percepción del impacto familiar”.

A continuación se describen los rasgos fundamentales de las cuatro categorías auxiliares, en función de la información expresada por los padres y de la triangulación realizada con la visión de los profesionales.

\subsection{Dominio personal de la condición de SD}

La presente categoría se refiere al modo en el que los padres afrontan la situación del síndrome de Down de su hijo, incluyendo la propia concepción que tienen del síndrome, la información sobre el mismo y las perspectivas de futuro que tienen sobre la situación futura de su hijo.

Todos los consultados coinciden en señalar cómo el nacimiento de un hijo con SD ha supuesto una transformación en la visión previa del propio concepto del síndrome. Si con anterioridad al nacimiento de su hijo, y aún durante los primeros momentos, las primeras voces que asocian a sus sentimientos son negativas (horror, pena, desgracia...), con el tiempo, el propio concepto de SD se desvanece de su mentalidad para dar paso únicamente al hijo que han tenido.

Es posible que la amplia información disponible sobre el SD ayude a esta evolución. Los padres afirman sentirse cómodos con el nivel de información que poseen y tanto ellos como los profesionales señalan cómo gracias a las nuevas tecnologías, y especialmente a Internet, el acceso a la información es cada vez más sencillo. Igualmente centros sanitarios, escolares y organizaciones dedicadas a la atención de personas con SD se consolidan como valiosas fuentes de información y recursos.

Respecto a las perspectivas sobre el futuro de sus hijos, puede verse una evolución desde una mayor preocupación por aspectos de la rutina diaria cotidiana en las primeras etapas del desarrollo hacia una mayor preocupación por el largo plazo, con una especial inquietud acerca de quién se hará cargo del hijo cuando los progenitores fallezcan. El futuro laboral y el nivel de independencia que alcanzarán los hijos en un futuro son dos de las cuestiones que suponen mayor preocupación para los padres. Los profesionales indican cómo estas expectativas dependen hondamente del nivel de realismo que los padres tienen sobre la discapacidad de sus hijos, señalando cómo en los casos en los que existe una distorsión entre la situación del hijo y la percepción de la misma por parte de sus padres, éstos tienden a tratar de compensar las limitaciones reales de sus hijos a través del anhelo de que éstos alcancen metas demasiado ambiciosas para sus circunstancias personales. 


\subsection{Vivencia personal y emocional}

Esta categoría hace referencia al impacto emocional que supone para los padres el síndrome de Down de su hijo. En este sentido, cabe destacar que el proceso de aceptación de la condición del hijo con síndrome de Down tiene una duración variable que sigue una trayectoria no lineal, dependiendo de la personalidad y carácter de cada padre. En opinión de los profesionales son pocos los casos en los que un padre muestra explícitamente ausencia de aceptación hacia su hijo con SD, manifestándose ésta, en el caso de existir, siempre de manera encubierta. Por otra parte, según indican los profesionales, una vez finalizado el proceso de aceptación del hijo, la vivencia y visión parental de cada uno de los padres resulta diferente, aunque los padres consultados no tienden a percibirlo de este modo. Los profesionales generalizan que, usualmente, es fácil detectar en las madres más gestos de sobreprotección mezclados con actitudes de exigencia hacia sus hijos, mientras que los padres demuestran posturas más despreocupadas, además de menos exigentes.

Para las familias creyentes, las convicciones religiosas constituyen un apoyo relevante en la aceptación y vivencia del SD de sus hijos. Sin embargo, la religión no es la única ni la principal red de apoyo con la que cuentan los padres, señalando tanto por padres como por profesionales la pareja, la familia y el colegio como algunos de los principales puntos de apoyo fundamentales de la vivencia parental.

\subsection{Experiencia familiar y social}

La categoría "experiencia familiar y social” hace referencia al modo en el que la presencia de un hijo con SD ha afectado a los vínculos familiares y hábitos sociales de la familia.

Respecto a la propia relación de pareja, padres y madres coinciden en considerar que su pareja no se ha visto afectada o ha supuesto, en cualquier caso, un fortalecimiento para la pareja. Tanto padres como profesionales coinciden al señalar que en el curso de una relación de pareja surgen distintos acontecimientos (un despido laboral, un fallecimiento, una enfermedad...) que suponen un reto y ponen a prueba la solidez de los vínculos de pareja, considerándose el SD como uno de estos acontecimientos imprevistos.

En los casos en los que existen hermanos, los padres indican que su intención es repartir la atención de forma equitativa entre todos sus hijos, aunque no siempre es posible debido a las exigencias derivadas de la condición de SD. Sin embargo, esto no repercute en un rechazo o antagonismo entre hermanos, sino que, por el contrario, la relación entre los mismos es calificada tanto por los padres como los profesionales de completamente normal. Ambos grupos de informantes aseveran que la presencia de un hermano con SD permite a los demás hijos adquirir de manera genuina una serie de valores que de otro modo alcanzarían mediante vías más artificiosas.

En relación al reparto de tareas en la pareja, los padres cuestionados consideran que la presencia del hijo con SD no ha supuesto una modificación concreta. En los casos

(C) Ediciones Universidad de Salamanca / CC BY-NC-ND

Siglo Cero, vol. 48 (2), n. ${ }^{\circ}$ 262, 2017, abril-junio, pp. 81-98 
en los que una de las dos partes se ocupa de manera principal de la crianza del hijo, ésta suele recaer sobre la madre. Sin embargo, los padres indican que esta división de tareas es de mutuo acuerdo y previo al nacimiento del hijo. Los profesionales señalan que los matrimonios más jóvenes tienden a llevar a cabo un reparto más equitativo de tareas, siendo más habitual que tanto el padre como la madre estén incorporados al mercado de trabajo en igualdad de condiciones, también en las familias con un hijo con SD.

En los casos analizados, los padres indican que la presencia de un hijo con SD no impide a la familia realizar actividades cotidianas, salvo en los casos en los que un plan supone una alteración brusca de la rutina familiar. Del mismo modo, los padres no detectan ningún tipo de impacto del hijo con SD en la relación con sus amistades.

Por último, es de destacar que los padres indican que, por lo general, no consideran a la sociedad preparada para aceptar sin prejuicios a las personas con SD, habiendo sido todos ellos testigos, en alguna ocasión, de situaciones desagradables (miradas indiscretas, comentarios inadecuados o risas despectivas). Sin embargo, ninguno de los padres y madres vivieron ninguna situación de rechazo directo hacia su hijo en su entorno próximo.

\subsection{Impacto económico}

Los padres indican cómo los gastos extra derivados del SD suponen un esfuerzo económico a pesar de que en España tanto la atención sanitaria como las necesidades básicas se encuentren cubiertas por el Estado. Sin embargo, algunos requerimientos derivados del SD deben ser costeados de forma directa y personal por los padres. En este sentido, los gastos que, en opinión de las familias consultadas, suponen un mayor esfuerzo económico son los gastos asociados a la escolarización del hijo, los tratamientos y terapias como logopedia, fisioterapia y tratamientos odontológicos y, por último, los servicios de ocio y actividades extraescolares. Como es natural, la opinión sobre la generación de gastos extra en la familia está estrechamente vinculada al poder adquisitivo de la misma, de manera que las familias con menor capacidad económica expresan un mayor descontento con la proporción de servicios que el Estado cubre de manera pública y gratuita, mientras que los grupos familiares de niveles económicos desahogados se sienten conformes y satisfechos con el tipo de atención cubierta por el Estado.

En cuanto a la vida laboral de los padres, tanto los padres como las madres indican que no ha sido necesario para ninguno de ellos realizar una modificación de su jornada laboral para atender a su hijo con SD. Sin embargo, reconocen que el SD implica que alguno de los miembros de la familia disponga de parte de su tiempo personal, y en ocasiones laboral, para satisfacer las necesidades de atención clínica-sanitaria o incluso burocrática que derivan de la propia discapacidad. 


\begin{tabular}{|c|c|c|c|}
\hline \multicolumn{4}{|c|}{ TABLA 3. Categorías, códigos, definición de los códigos y citas } \\
\hline Categoría & Código & DeFINICIÓN DEL CÓDIGO & CITA \\
\hline \multirow{3}{*}{$\begin{array}{l}\text { a) Dominio } \\
\text { personal } \\
\text { de la } \\
\text { condición } \\
\text { de SD }\end{array}$} & $\begin{array}{l}\text { C1. Concepción } \\
\text { personal }\end{array}$ & $\begin{array}{l}\text { Conjunto de nociones con las que } \\
\text { el individuo identifica el SD. }\end{array}$ & $\begin{array}{c}\text { M1 - 1:22 [Para mí, antes de nacer mi } \\
\text { hija era un horror. Ya se me ha pasado. } \\
\text { Ya no es un horror. Es una minusva- } \\
\text { lía.] (37:37) }\end{array}$ \\
\hline & C2. Información & $\begin{array}{l}\text { Nivel de información y conoci- } \\
\text { miento sobre el SD. }\end{array}$ & $\begin{array}{l}\text { P3 - 6:9 [No sé si el tener más conoci- } \\
\text { miento sobre el propio estado del SD } \\
\text { a mí me daría más facilidad de tratar a } \\
\text { mi hijo. Para nada.] (40:40) }\end{array}$ \\
\hline & $\begin{array}{l}\text { C3. Expectativas } \\
\text { de futuro }\end{array}$ & $\begin{array}{l}\text { Perspectiva y visión personal sobre } \\
\text { la situación futura del hijo con SD. }\end{array}$ & $\begin{array}{c}\text { P1 - 4:25 [Que sea una niña feliz.] } \\
(70: 70)\end{array}$ \\
\hline \multirow{5}{*}{$\begin{array}{l}\text { b) Vivencia } \\
\text { personal y } \\
\text { emocional }\end{array}$} & $\begin{array}{l}\text { C4. La primera } \\
\text { noticia }\end{array}$ & $\begin{array}{l}\text { Momento de comunicación de } \\
\text { diagnóstico a los padres. }\end{array}$ & M3 - 3:11 [Fue un shock.] (162:162) \\
\hline & $\begin{array}{l}\text { C5. Experiencias } \\
\text { emocionales }\end{array}$ & $\begin{array}{c}\text { Vivencias y sentimientos personales } \\
\text { sobre el SD. }\end{array}$ & $\begin{array}{l}\text { P1 - 4:6 [Cogí un cabreo bastante gor- } \\
\text { do. Pero bueno, se me pasó.] (176:176) }\end{array}$ \\
\hline & $\begin{array}{l}\text { C6. Proceso de } \\
\text { aceptación }\end{array}$ & $\begin{array}{c}\text { Sentimientos y situaciones relacio- } \\
\text { nados con la aceptación personal } \\
\text { del hijo. }\end{array}$ & $\begin{array}{l}\text { M1 - 1:43 [Pero lo acepté. En algún } \\
\text { momento lo acepté.] (379:379) }\end{array}$ \\
\hline & C7. Apoyo & $\begin{array}{c}\text { Recursos personales o institucio- } \\
\text { nales que prestan soporte personal } \\
\text { o familiar. }\end{array}$ & $\begin{array}{l}\text { M1 - 1:29 [O sea, me apoyo mucho en } \\
\text { ellos, en el colegio...] (513:513) }\end{array}$ \\
\hline & C8. Religión & $\begin{array}{l}\text { Conjunto de creencias y valores } \\
\text { espirituales personales. }\end{array}$ & $\begin{array}{c}\text { P3 - 6:15 [Encuentro apoyo en mis } \\
\text { valores.] (349:349) }\end{array}$ \\
\hline \multirow{6}{*}{$\begin{array}{l}\text { c) Experiencia } \\
\text { familiar y } \\
\text { social }\end{array}$} & C9. Pareja & $\begin{array}{l}\text { Aspectos relacionados con la vida } \\
\text { en pareja. }\end{array}$ & $\begin{array}{c}\text { M3 - 3:17 [Es que me parece que mi } \\
\text { vida no ha cambiado nada. Que sería } \\
\text { la misma.] (205:205) }\end{array}$ \\
\hline & C10. Hermanos & $\begin{array}{l}\text { Percepción personal de las vivencias } \\
\text { de los hermanos del hijo con SD. }\end{array}$ & $\begin{array}{c}\text { P3 - 6:17 [Mi hijo siempre ha tratado a } \\
\text { su hermano (con SD) de la manera más } \\
\text { normal y más natural como lo hace- } \\
\text { mos todos en casa.] (441:441) }\end{array}$ \\
\hline & $\begin{array}{l}\text { C11. Familia } \\
\text { directa }\end{array}$ & $\begin{array}{l}\text { Reacción y vivencias de los familia- } \\
\text { res respecto al SD. }\end{array}$ & $\begin{array}{c}\text { M2 - 2:18 [Le quieren mucho y está } \\
\text { muy aceptado, no hay ningún proble- } \\
\text { ma.] (796:796) }\end{array}$ \\
\hline & $\begin{array}{l}\text { C12. Dinámica } \\
\text { familiar }\end{array}$ & Roles familiares y planes en familia. & $\begin{array}{c}\text { P3 - 6:20 [Todos los roles son compar- } \\
\text { tidos.] (481:481) }\end{array}$ \\
\hline & C13. Amigos & $\begin{array}{c}\text { Experiencias y reacciones de amigos } \\
\text { cercanos a la familia. }\end{array}$ & $\begin{array}{c}\text { P2 - 5:16 [Amistades más íntimas } \\
\text { no hacen ningún tipo de diferencia.] } \\
\text { (502:502) }\end{array}$ \\
\hline & C14. Vida social & $\begin{array}{l}\text { Nivel de participación en la vida } \\
\text { social en relación con tener un hijo } \\
\text { con SD. }\end{array}$ & $\begin{array}{l}\text { M1 - 1:47 [Me aparté más] (928:928); } \\
\text { 1:48 [Porque entre que tenía una bue- } \\
\text { na dosis de cosas que hacer y no tenía } \\
\text { muchas ganas de dar explicaciones, ni } \\
\quad \text { de contar...] (928:928) }\end{array}$ \\
\hline
\end{tabular}


TABLA 3. Categorías, códigos, definición de los códigos y citas

\begin{tabular}{|c|c|c|c|}
\hline CATEgoría & CóDIGO & Definición DEL Código & CITA \\
\hline $\begin{array}{c}\text { c) Experiencia } \\
\text { familiar y } \\
\text { social }\end{array}$ & C15. Sociedad & $\begin{array}{c}\text { Percepciones personales de cómo la } \\
\text { sociedad actúa ante el SD del hijo. }\end{array}$ & $\begin{array}{c}\text { M1 - 1:17 [No todo el mundo es bue- } \\
\text { no. Y están indefensos.] (137:137); 1:18 } \\
\text { [Miradas de estas que abrasan, que van } \\
\text { como riéndose...] (187:187) }\end{array}$ \\
\hline \multirow{2}{*}{$\begin{array}{c}\text { d) Impacto } \\
\text { económico }\end{array}$} & $\begin{array}{c}\text { C16. Gastos } \\
\text { adicionales }\end{array}$ & $\begin{array}{c}\text { Conjunto de gastos extra que pro- } \\
\text { voca el SD en la familia. }\end{array}$ & $\begin{array}{c}\text { P2 - 5:17 [Depende de la situación que } \\
\text { tiene cada uno.] (568:568) }\end{array}$ \\
\cline { 2 - 4 } & C17. Vida laboral & $\begin{array}{c}\text { Factores relacionados con la carrera } \\
\text { laboral personal. }\end{array}$ & $\begin{array}{c}\text { M3 - 3:27 [Hay un montón de actos } \\
\text { burocráticos, es que no me sale la } \\
\text { palabra, que tienes que dejar de ir a } \\
\text { trabajar para hacerlos...] (817:817) }\end{array}$ \\
\hline
\end{tabular}

\subsection{Teoría fundamentada generada}

El análisis de las categorías seleccionadas, y de los códigos que las conforman, ha permitido la elaboración de una teoría fundamentada que agrupa los mismos en la percepción del impacto familiar según los propios padres. La parentalidad de un hijo con SD supone un reto para los padres y los demás miembros de la familia, así como en la estructura y dinámica del sistema familiar en función de múltiples factores. Los impactos generados a partir de este hecho en la familia son tanto positivos como negativos, pero el efecto positivo sobre la dinámica familiar parece ser preponderante. La Figura 1 muestra la red categórica de relaciones originadas en la teoría generada, así como el tipo de impacto de cada uno de los términos fundamentales, señalando los principales conceptos que se integran en cada una de las categorías determinadas y su interacción.

\section{Discusión}

Los resultados sugieren la existencia de impacto en niveles heterogéneos, sobre diferentes dimensiones del ámbito familiar originado por el nacimiento de un hijo con SD. Si bien, cada situación familiar debe ser considerada de forma independiente, este estudio ha permitido una aproximación a la percepción en primera persona de la situación real de familias contemporáneas viviendo una situación de discapacidad en su seno.

\subsection{Mi bijo tiene sindrome de Down. La experiencia de la parentalidad}

Los resultados de la presente investigación corroboran estudios previos que sugieren que la parentalidad de un hijo con síndrome de Down aporta tanto cohesión, como experiencias y valores positivos al sistema familiar (Choi y Riper, 2016; Nelson 
FIGURA 1. Teoría generada a partir del análisis de las entrevistas parentales y profesionales sobre el impacto familiar del SD (Fuente: Autor, 2017)

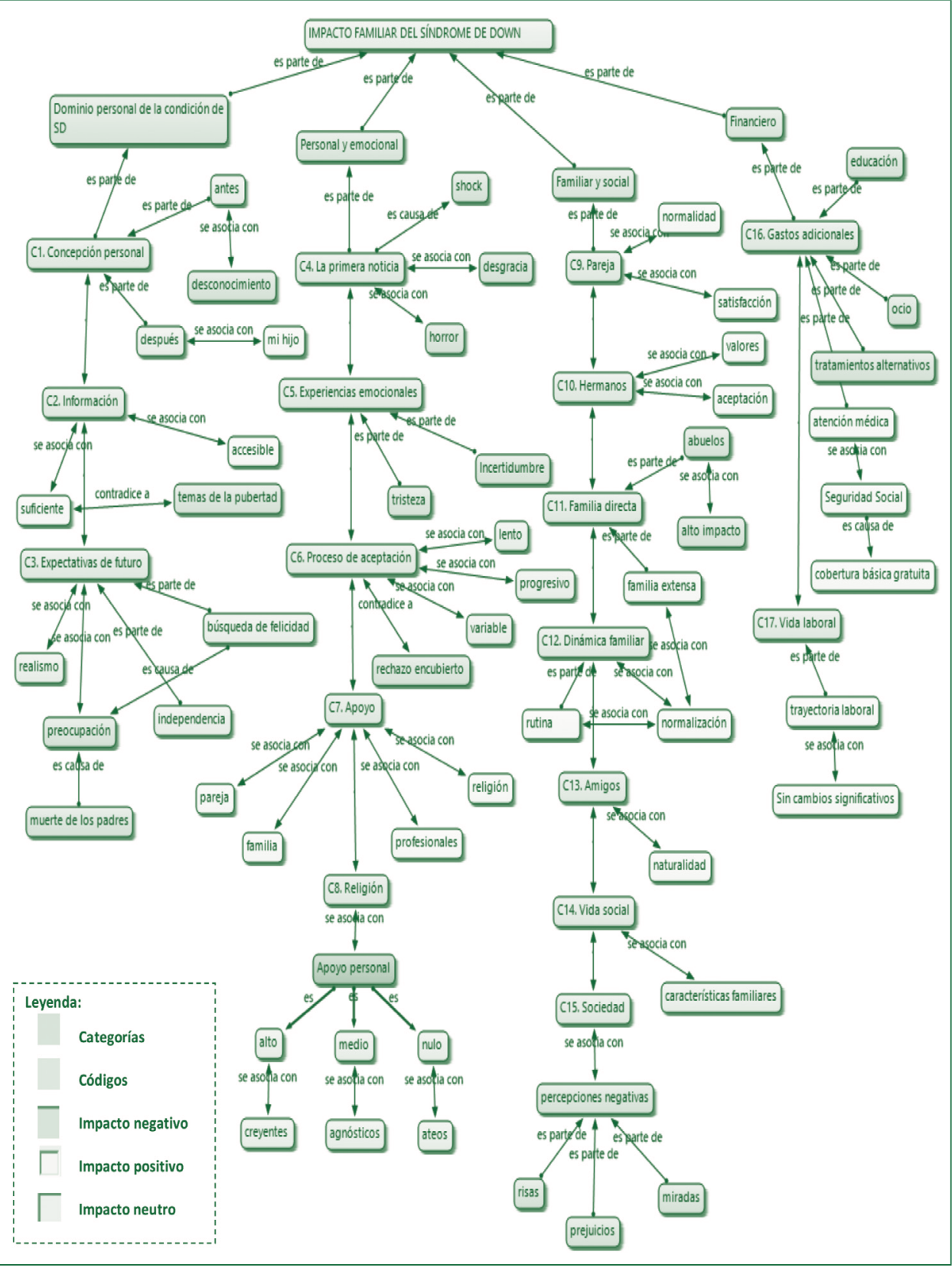

(C) Ediciones Universidad de Salamanca / CC BY-NC-ND

Siglo Cero, vol. 48 (2), n. ${ }^{\circ}$ 262, 2017, abril-junio, pp. 81-98

$$
-91-
$$


et al., 2016; Skotko, Levine y Goldstein, 2011a; Skotko, Levine, Macklin y Goldstein, 2015). La sensación percibida en las familias hacia su situación fue de normalidad, observando que, a pesar de haber acogido la noticia del SD de sus hijos como un evento imprevisto e impactante, desarrollaron las estrategias necesarias para afrontar la situación de manera resolutiva, a través de la adquisición de habilidades cohesivas de afrontamiento familiar. Actualmente, estas familias se autoperciben como grupos familiares de desarrollo típico, envueltos en una rutina caracterizada por la normalidad. Esta sensación de normalidad, ya percibida por Cunningham (1996) en las familias de niños con SD, engloba a los diferentes miembros que conforman la familia extensa.

Conyugalmente, los padres y madres entrevistados han manifestado altos índices de cohesión y apoyo entre los dos miembros de la pareja, descartando la idea de la discapacidad como argumento de problemas maritales o causa de divorcio. A pesar de que la comunicación de un diagnóstico de discapacidad pudo haber sido asumida como un evento estresor puntual al que la pareja tuvo que hacer frente en un momento concreto de su ciclo vital, ambos miembros de la pareja han puntualizado que el SD de sus hijos ha llegado incluso a fomentar la creación de un vínculo más sólido entre ambos, al haber sabido superar juntos y con éxito un desafío imprevisto. Dentro de la dinámica de la pareja, el reparto de tareas entre sus miembros es percibido como justo y equitativo. No obstante, es necesario hacer hincapié en las diferencias encontradas entre la perspectiva de los padres y la de los profesionales. En consonancia con Pereira-Silva et al. (2015), los profesionales consultados en la presente investigación señalan que, a pesar de existir actualmente un incremento del porcentaje de tareas familiares compartidas equitativamente por los miembros de la familia, las responsabilidades derivadas de la crianza de los hijos sigue asumiéndose de forma mayoritaria por las madres, en comparación a los padres.

Retomando la línea de percepciones positivas, los padres observaron, asimismo, actitudes positivas de sus otros hijos respecto a sus primogénitos con SD. En consonancia con otros estudios, los padres advirtieron que sus hijos sin discapacidad poseen un buen autoconcepto de sí mismos y de sus hermanos (Graff et al., 2012; Skotko y Levine, 2006) y que, inclusive, han desarrollado, a partir de la situación vivida, un bagaje de valores humanos altamente beneficioso para sí mismos (Cuskelly, 2016; Mandleco y Webb, 2015; Skotko, Levine y Goldstein, 2011b; Skotko et al., 2015). La relación entre hermanos percibida por la muestra analizada (tanto padres como profesionales) fue buena y satisfactoria, no encontrando casos concretos de rechazo implícito ni explícito por parte de hermanos sin discapacidad hacia hermanos con SD.

Este estudio pone de manifiesto, así mismo, la importancia que los sistemas y fuentes de apoyo (especialmente, pareja, familia, profesionales y creencias religiosas) poseen en relación al sistema familiar, corroborando el valor fundamental que los cónyuges otorgan a la pareja, con la cual han asegurado coincidir en las opiniones respecto a la crianza de su hijo y a la cual han considerado pilar central de apoyo. No obstante, una de las principales diferencias encontradas en el análisis de los discursos de los padres y de los expertos fue la existencia de cierto desequilibrio, percibido por los profesionales, entre la visión del padre y de la madre sobre la situación del hijo, el estilo de crianza y el reparto de tareas. Estos resultados se apoyan en investigaciones 
como la llevada a cabo por Povee, Roberts, Bourke y Leonard (2012), quienes observaron la existencia de diferencias significativas entre el estilo educativo en los padres que conlleva el surgimiento de pequeños enfrentamientos entre ambos.

Por su parte, los sistemas de creencias y valores espirituales funcionan como una fuente fundamental de sostén, siempre y cuando los padres se muestren abiertos a ellos (Nelson et al., 2016). La presente investigación coincide con la idea de que las personas que manifiestan una fe profunda encuentran en la religión un pilar al que agarrarse y vivir con tranquilidad su día a día. Mientras que para los padres y madres creyentes entrevistados la religión jugó un papel decisivo a la hora de aceptar el diagnóstico del hijo, para los padres no creyentes este proceso de aceptación se sustentó en otro tipo de recursos personales.

En términos generales, la dinámica general observada en las familias con un hijo con SD fue de normalidad, permitiendo que el grupo familiar haga naturalmente su rutina diaria, sin tener que realizar grandes cambios exprofeso por su hijo con SD.

\subsection{Los retos de la parentalidad del SD. Dimensiones con mayor impacto}

El presente estudio coincide con las investigaciones que sostienen la idea de que el nacimiento de un hijo con discapacidad se constituye como un evento inesperado, que impacta profundamente en los padres causando un desequilibrio inicial en la estructura familiar, como consecuencia de la divergencia frente a la idealidad de las expectativas parentales (Greiner y Conklin, 2015). Padres, madres y profesionales explicaron que el momento de la primera noticia fue vivido con sorpresa y confusión, siendo la emoción más referida la tristeza.

Así mismo, la crianza de un niño con SD genera incertidumbre en los padres que inicialmente desconocen las futuras necesidades de su inesperado bebé. Si bien es cierto que los padres de esta investigación manifestaron satisfacción con el nivel de información habida, así como con la facilidad de acceso a la misma en caso de necesidad, señalaron que suelen presentar dudas a la hora de resolver cuestiones acerca de temas de desarrollo en la adolescencia y aspectos de la reproducción sexual. Los padres afirmaron que la adolescencia de sus hijos con discapacidad es una de las etapas ante la que sienten mayor inseguridad a la hora de decidir y actuar, debido a que no cuentan con la información necesaria de poblaciones atípicas para solventar sus dudas. La red social y la profesional se constituyen en este período como dos de los pilares fundamentales de apoyo para la familia.

Al hablar de parentalidad es preciso recalcar que el hecho de la crianza de un hijo no sólo incide de manera directa sobre los padres, sino que posee diversas repercusiones en los demás miembros de la familia (Rooke y Pereira-Silva, 2016). El aspecto más relevante del impacto del SD en la familia extensa, mencionado por todos los padres y madres, fue el impacto que el diagnóstico de su hijo tiene sobre los abuelos. El impacto de una circunstancia de discapacidad en los nietos les supone consternación y conmoción, así como la necesidad de recomponer drásticamente las expectativas que habían conjeturado con ilusión para sus nietos. Tres son los factores que los padres 
y las madres de este estudio han atribuido a la dificultad en la aceptación del cuadro diagnóstico de un nieto: la avanzada edad de sus padres, la situación y perspectiva negativista de la discapacidad existente en la época de sus padres y el desconocimiento de casos reales, así como de información concreta, relacionados con el cuadro diagnóstico. Las familias coinciden con Miller, Buys y Woodbridge (2012) en señalar la necesidad expresada por los abuelos de poseer mejores recursos y apoyos relacionados con lo que les sucede a sus nietos, así como establecer contacto con otros abuelos o familias que se encuentran en la misma situación que ellos. No obstante, las familias indicaron que, superada la etapa inicial de shock, los abuelos se consolidan como uno de los principales apoyos parentales en lo relacionado con la crianza de un hijo con discapacidad, estableciendo estrechos y fuertes vínculos afectivos con sus nietos.

La crianza de un hijo con SD, así como la calidad de vida familiar e individual, se encuentran íntimamente ligadas al estatus económico de la familia, vinculándose, por tanto, de forma directa la capacidad económica con la accesibilidad a unos u otros recursos. Esta idea queda igualmente reflejada tanto en el presente estudio como en las investigaciones llevadas a cabo por Foley et al. (2014) y Haimour y Abu-Hawwash (2012). Es este aspecto, es preciso recalcar que, a pesar de que España goza de una amplia cobertura sanitaria gratuita y universal a través de la Seguridad Social, las familias de este estudio confirmaron la necesidad de tener que acudir a tratamientos adicionales, como logopedia, fisioterapia u odontología o recurrir a servicios de ocio y tiempo libre especializados, que suponen una fuerte demanda económica para el sistema familiar.

Otro gran reto que manifestaron los padres a la hora de vivir con la experiencia del SD se vincula con los prejuicios y actitudes negativos exhibidos mayoritariamente por terceras personas, ajenas a sus familias. Reacciones ofensivas hacia sus hijos con SD por parte de la sociedad, que actualmente se considera avanzada y progresista en referencia al pensamiento social, fueron causa de daños emocionales en los padres. Flórez (2012) recordó en su estudio que no resulta tan complicado hoy en día percibir este tipo de actitudes, a veces muy poco sutiles, que provocan en las familias inevitables sentimientos de dolor y frustración.

Para finalizar, en relación a las expectativas parentales a largo plazo concernientes a las personas con SD existió un consenso implícito. Homogéneamente, todos los padres de este estudio mostraron altos índices de preocupación a la hora de hablar del futuro de sus hijos, coincidiendo en la necesidad de buscar un lugar de confianza en el que sus hijos puedan vivir cuando ellos fallezcan. Para favorecer el alcance de estas expectativas, los padres tienden a desarrollar planes de acción, concentrados en ofrecer a sus hijos la mayor cantidad de estrategias posibles que fomenten su autonomía e independencia. Todos los padres compartieron la misma idea de que sus hijos puedan optar a trabajar en puestos especializados y adaptados a las características individuales de cada uno de ellos, siendo su última prioridad la garantía de que sus hijos se encuentren en un futuro atendidos por personas morales y honradas. Los profesionales entrevistados, en consonancia con investigaciones actuales (Whittingham, Wee, Sanders y Boyd, 2013), hicieron hincapié en el estrecho vínculo y dependencia existente entre el cumplimiento de las expectativas parentales y la situación real del hijo. Cuanto mayor 
nivel de realismo aporta la visión parental, más sencillo es encontrar una solución adecuada evitando situaciones dominadas por la frustración emocional.

\section{Limitaciones y futuras líneas de investigación}

La presente investigación se encuentra sujeta a diversas limitaciones, entre las que se destaca el sesgo en la selección de la muestra, así como su tamaño reducido. La muestra representada en este estudio no corresponde a la población base ni a la familia típica de la realidad española actual, dado que toda la muestra a la que se ha tenido oportunidad de acceso ha sido seleccionada únicamente por criterios de accesibilidad en un mismo contexto. Las características familiares descritas en este artículo tienden a la homogeneidad de una realidad particular. Este hecho impide que se puedan generalizar las conclusiones obtenidas a todas las familias que tienen hijos con síndrome de Down en España.

Así mismo, sería conveniente dedicar futuras líneas de investigación a explorar el impacto del SD en familias provenientes de otro tipo de contextos socioculturales y económicos españoles, extendiendo incluso el ámbito de la investigación a realizar comparaciones entre el impacto del SD a nivel de familias de origen rural y urbano. Además, siendo conscientes de que las circunstancias de las familias que viven en $\mathrm{SD}$ en su seno presentan características totalmente divergentes en países diferentes al nuestro, se sugiere una línea de investigación futura en el análisis de comparaciones interculturales que amplíen el conocimiento global de la situación actual e internacional de la vivencia del SD en el seno familiar.

\section{Agradecimientos}

Nos gustaría mostrar nuestro agradecimiento al Colegio de Educación Especial Cambrils por abrir sus puertas a este proyecto; a Nacho Martín Blasco por facilitarnos la realización de la presente investigación, así como el acceso a la muestra; a Nuria Campillos por su profesionalidad a la hora de realizar las entrevistas; a la Fundación Prodis y a Yone Castro por su constante apoyo, así como por su asesoramiento durante el proceso de investigación y análisis de los datos, y, especialmente, a todos los participantes en el presente estudio, familias y profesionales del campo de la educación especial, que han querido compartir con nosotros la información que conforma el cuerpo de la presente investigación.

\section{Referencias bibliográficas}

Adamson, L. B., Deckner, D. F. y Bakeman, R. (2010). Early interests and joint engagement in typical development, autism and Down syndrome. Journal of Autism and Developmental Disorders, 40, 665-676. doi: 10.1007/s10803-009-0914-1. 
Albuquerque, S., Pereira, M., Fonseca, A. y Canavarro, C. (2012). Impacto familiar e ajustamento de pais de crianças com diagnóstico de anomalia congênita: influência dos determinantes da criança. Revista de Psiquiatría Clínica, 39, 136-141.

Almasri, N. A., Palisano, R. J., Dunst, C. J., Chiarello, L. A., O’Neil, M. E. y Polansky, M. (2011). Determinants of needs of families of children and youth with cerebral palsy. Children's Health Care, 40 (2), 130-154. doi: 10.1080/02739615.2011.564568.

Arumugam, A., Raja, K., Venugopalan, M., Chandrasekaran, B., Kovanur, K., Muthusamy, H. y Shanmugam, N. (2015). Down syndrome: A narrative review with a focus on anatomical features. Clinical Anatomy, 29, 568-577. doi: 10.1002/ca.22672.

Choi, H. y Riper, M. V. (2016). Maternal perceptions to open-ended questions about life with Down syndrome in Korea. The Qualitative Report, 21 (2), 288-298.

Collins, T. y Coughlan, B. (2016). Experiences of mothers in Romania after hearing from medical professionals that their child has a disability. Journal of Policy and Practice in Intellectual Disabilities, 13 (1), 4-14. doi:10.1111/jppi.12141.

Cunningham, C. (1996). Families of children with Down syndrome. Down Syndrome Research and Practice, 4 (3), 87-95.

Cuskelly, M. (2016). Contributors to adult sibling relationships and intention to care of siblings of individuals with Down syndrome. American Journal on Intellectual and Developmental Disabilities, 121 (3), 204-218. doi: 10.1352/1944-7558-121.3.204.

Durmaz, A., Cankaya, T., Durmaz, B., Vahabi, A., Gunduz, C., Cogulu, O. y Ozkinay, F. (2011). Interview with parents of children with Down syndrome: their perceptions and feelings. The Indian Journal of Pediatrics, 78 (6), 698-702. doi: 10.1007/s12098-010-0315-7.

FARBER, B. y KIRK, S. A. (1959). Effects of a severely mentally retarded child on family integration. Monographs of the Society for Research in Child Development, 24, 1-112.

Federación Estatal de Asociaciones de Profesionales de la Atención Temprana-Gat (2011). Mejora del proceso de comunicación del diagnóstico de trastorno en el desarrollo $o$ discapacidad en la primera infancia. La primera noticia. Madrid: Real Patronato sobre Discapacidad.

FlóreZ, J. (2012). Actitudes y mentalidades de la sociedad ante el síndrome de Down. Revista Sindrome de Down, 29, 65-69.

Foley, K. R., Girdler, S., Downs, J., Jacoby, P., Bourke, J., Lennox, N., Einfeld, S., Llewellyn, G., Parmenter, T. R. y Leonard, H. (2014). Relationship between family quality of life and day occupations of young people with Down syndrome. Social Psychiatry and Psychiatric Epidemiology, 49 (9), 1455-1465. doi: 10.1007/s00127-013-0812-x.

Gath, A. (1977). The impact of an abnormal child upon the parents. British Journal of Psychiatry, 130, 405-410.

Glidden, L. M., Bamberger, K. T., Turek, K. C. y Hill, K. L. (2010). Predicting mother/ father-child interactions: parental personality and well-being, socioeconomic variables and child disability status. Journal of Applied Research in Intellectual Disabilities, 23 (1), 3-13. doi: 10.1111/j.1468-3148.2009.00549.x.

Graff, C., Mandleco, B., Dyches, T., Coverston, C., Roper, S. y Freeborn, D. (2012). Perspectives of adolescent siblings of children with Down syndrome who have multiple health problems. Journal of Family Nursing, 18 (2), 175-199. doi: 10.1177/1074840712439797.

GreEN, S. E. (2007). "We're tired, not sad": benefits and burdens of mothering a child with a disability. Social Science and Medicine, 64 (1), 150-163. doi: 10.1016/j.socscimed.2006.08.025.

Greiner, A. L. y Conklin, J. (2015). Breaking bad news to a pregnant woman with a fetal abnormality on ultrasound. Obstetrical and Gynecological Survey, 70 (1), 39-44. doi: 10.1097/ ogx.0000000000000149.

(c) Ediciones Universidad de Salamanca / CC BY-NC-ND

Siglo Cero, vol. 48 (2), n. ${ }^{\circ}$ 262, 2017, abril-junio, pp. 81-98

$$
-96-
$$


Haimour, A. I. y Abu-Hawwash, R. M. (2012). Evaluating quality of life of parents having a child with disability. International Interdisciplinary Journal of Education, 1 (2), 37-43.

Hastings, R. P. y Taunt, H. M. (2002). Positive perceptions in families of children with developmental disabilities. American Journal on Mental Retardation, 107 (2), 116-127. doi: 10.1352/0895-8017(2002)107<0116:PPIFOC>2.0.CO;2.

Helff, C. M. y Glidden, L. M. (1998). More positive or less negative? Trends in research on adjustment of families rearing children with developmental disabilities. Mental Retardation, 36 (6), 457-464.

Hornby, G. (1995). Effects on fathers of children with Down syndrome. Journal of Child and Family Studies, 4 (2), 239-255.

Kramm, E. R. (1963). Families of mongoloid children. Washington D. C.: Government Printing Office.

Lancet Neurology, The (2016). Dementia in Down's syndrome: still much to learn. The Lancet Neurology, 15 (8), 775. doi: 10.1016/S1474-4422(16)30082-5.

LANFRAnChi, S. y Vianello, R. (2012). Stress, locus of control, and family cohesion and adaptability in parents of children with Down, Williams, Fragile X, and Prader-Willi syndromes. American Journal on Intellectual and Developmental Disabilities, 117 (3), 207-224. doi: 10.1352/1944-7558-117.3.207.

Migerode, F., Maes, B., Buysse, A. y Brondeel, R. (2012). Quality of life in adolescents with a disability and their parents: the mediating role of social support and resilience. Journal of Developmental and Physical Disabilities, 24 (5), 487-503. doi: 10.1007/s10882-012-9285-1.

Miller, E., Buys, L. y WoOdbridge, S. (2012). Impact of disability on families: grandparents' perspectives. Journal of Intellectual Disability Research, 56 (1), 102-110. doi: 10.1111/j.13652788.2011.01403.x.

Mitchell, D. B., Hauser-Cram, P. y Crossman, M. K. (2015). Relationship dimensions of the "Down syndrome advantage". Journal of Intellectual Disability Research, 59 (6), 506-518. doi: $10.1111 /$ jir.12153.

Mullins, D., Daly, E., Simmons, A., Beacher, F., Foy, C. M., Lovestone, S., Hallahan, B., Murphy, K. C. y Murphy, D. G. (2013). Dementia in Down's syndrome: an MRI comparison with Alzheimer's disease in the general population. Journal of neurodevelopmental disorders, 5 (19), 1-13. doi: 10.1186/1866-1955-5-19.

Nelson, B. S., Monk, J. K., Malone, J., Staats, N., Tanner, A. y Springer, N. P. (2016). Comparing parents of children with Down syndrome at different life span stages. Journal of Marriage and Family, 78 (4), 1131-1148. doi: 10.1111/jomf.12312.

Pereira-Silva, N. L., Oliveira, L. y Rooke, M. (2015). Famílias com adolescente com síndrome de Down: apoio social e recursos familiares. Avances en Psicología Latinoamericana, 33 (2), 269-283. doi: 10.12804/ap133.02.2015.07.

Povee, K., Roberts, L., Bourke, J. y Leonard, H. (2012). Family functioning in families with a child with Down syndrome: a mixed methods approach. Journal of Intellectual Disability Research, 56 (10), 961-973. doi: 10.1111/j.1365-2788.2012.01561.x.

Roizen, N. y Patterson, D. (2003) Down's syndrome. The Lancet, 361 (9365), 1281-1289. doi: 10.1016/S0140-6736(03)12987-X.

Rooke, M. I. y Pereira-Silva, N. L. (2016). Indicativos de resiliência familiar em famílias de crianças com síndrome de Down. Estudos de piscología (Campinas), 33 (1), 117-126. doi: 10.1590/1982-027520160001000012-.

Schonell, F. J. y WatTs, B. H. (1956). A first survey of the effects of a subnormal child on the family unit. American Journal of Mental Deficiency, 61, 210-219.

() Ediciones Universidad de Salamanca / CC BY-NC-ND

Siglo Cero, vol. 48 (2), n. ${ }^{\circ}$ 262, 2017, abril-junio, pp. 81-98 
Sherman, S. L., Allen, E. G., Bean, L. H. y Freeman, S. B. (2007). Epidemiology of Down syndrome. Mental Retardation and Developmental Disabilities Research Reviews, 13 (3), 221-227.

Sкотко, B. G. y Levine, S. P. (2006). What the other children are thinking: brothers and sisters of persons with Down syndrome. American Journal of Medical Genetics, 142C, 180-186.

Sкотко, B. G., Levine, S. P. y Goldstein, R. (2011a). Having a son or daughter with Down syndrome: perspectives from mothers and fathers. American Journal of Medical Genetics Part A, 155 (10), 2335-2347. doi: 10.1002/ajmg.a.34293.

Sкотко, B. G., Levine, S. P. y Goldstein, R. (2011b). Having a brother or sister with Down syndrome: perspectives from siblings. American Journal of Medical Genetics Part A, 155 (10), 2348-2359. doi: 10.1002/ajmg.a.34228.

Skotko, B. G., Levine, S. P., Macklin, E. A. y Goldstein, R. D. (2015). Family perspectives about Down syndrome. American Journal of Medical Genetics Part A, 9999A, 1-12. doi: 10.1002/ajmg.a.37520.

Stein, R. E. K. y Riessman, C. K. (1980). The development of the Impact on Family Scale: preliminary findings. Medical Care, 18, 465-472.

Turnbull, A. P. y Turnbull, H. R. (2001). Families, professionals and exceptionality: collaborating for empowerment (4. ${ }^{\mathrm{a}}$ ed.). Upper Saddle River: Prentice Hall.

VOn BerTalanfFy, L. (1968). General systems theory: foundations, development, applications. Nueva York: George Braziller.

Whittingham, K., Wee, D., Sanders, M. R. y Boyd, R. (2013). Sorrow, coping and resiliency: parents of children with cerebral palsy share their experiences. Disability and rehabilitation, 35 (17), 1447-1452.

Woodbridge, S., Buys, L. y Miller, E. (2011). 'My grandchild has a disability': impact on grandparenting identity, roles and relationships. Journal of Aging Studies, 25 (4), 355-363. doi: 10.1016/j.jaging.2011.01.002. 